\title{
Adherent Primary Cultures of Mouse Intercostal Muscle Fibers for Isolated Fiber Studies
}

\author{
Patrick Robison, Erick O. Hernández-Ochoa, and Martin F. Schneider \\ Department of Biochemistry and Molecular Biology, University of Maryland School of Medicine, 108 N. Greene Street, \\ Baltimore, MD 21201, USA \\ Correspondence should be addressed to Erick O. Hernández-Ochoa, ehern001@umaryland.edu
}

Received 26 April 2011; Accepted 24 May 2011

Academic Editor: Robert J. Bloch

Copyright ( 92011 Patrick Robison et al. This is an open access article distributed under the Creative Commons Attribution License, which permits unrestricted use, distribution, and reproduction in any medium, provided the original work is properly cited.

\begin{abstract}
Primary culture models of single adult skeletal muscle fibers dissociated from locomotor muscles adhered to glass coverslips are routine and allow monitoring of functional processes in living cultured fibers. To date, such isolated fiber cultures have not been established for respiratory muscles, despite the fact that dysfunction of core respiratory muscles leading to respiratory arrest is the most common cause of death in many muscular diseases. Here we present the first description of an adherent culture system for single adult intercostal muscle fibers from the adult mouse. This system allows for monitoring functional properties of these living muscle fibers in culture with or without electrical field stimulation to drive muscle fiber contraction at physiological or pathological respiratory firing patterns. We also provide initial characterization of these fibers, demonstrating several common techniques in this new model system in the context of the established Flexor Digitorum Brevis muscle primary culture model.
\end{abstract}

\section{Introduction}

The bodies of vertebrates include hundreds of skeletal muscles, each involved in performing different motor tasks at specific anatomical locations. This variety in skeletal muscles and their functions implies genotypic and phenotypic diversity among skeletal muscle fibers. In fact, the preferential expression of different muscular protein isoforms and therefore the existence of distinct muscle fiber phenotypes is one of the main determinants of the muscle performance $[1,2]$. Not only does this diversity manifest in multiple muscle groups but also a single muscle expresses heterogeneous populations of slow and fast-type muscle fibers [1,3]. Excitationcontraction coupling properties, the sensitivity of the contractile apparatus to $\mathrm{Ca}^{2+}$, mechanical power output, shortening velocity, and rate of ATP hydrolysis are also known to vary greatly from fiber to fiber $[1,4-7]$. The intercostal muscles are an exemplary case of such a heterogeneous muscle population, making them an attractive model for comparative studies.

The mechanical functions of the intercostal muscles during ventilation are highly complex. There are two major division criteria for intercostal fibers: anatomical and functional. Anatomical divisions are based on location on the internal or external side of the ribs; functional divisions are based on participation in expiratory or inspiratory respiration [8]. The external intercostals and the parasternal intercostals function in inspiratory breathing, whereas the internal intercostals have an expiratory function [9]. Although the function of the diaphragm appears to dominate breathing at rest [10], the intercostal muscles may contribute c.a. $40 \%$ of the volume shift by movement of the thoracic wall [11]. During intensified breathing, the contribution of the intercostals becomes more prominent $[12,13]$. The lateral internal intercostals are also recruited for a variety of nonventilatory functions including the cough reflex and speech as well as for postural support [10].

Previous work estimates that about $60 \%$ of the intercostal fibers are slow-type and $40 \%$ fast-type fibers $[14,15]$. As a result of this diversity, the same preparation can be used to address many aspects of muscle fiber-type physiology. Also, due to their involvement in the mechanics of ventilation and the fact that respiratory pathology is common in several diseases (e.g., muscular dystrophy, chronic obstructive 
pulmonary disease, amyotrophic lateral sclerosis, hereditary polyneuropathies, autoimmune conditions like myasthenia gravis, muscular paralysis, etc.), intercostal muscles represent a model of high clinical relevance.

Indeed, in cases where diagnosis and treatment for muscular dystrophy is available, respiratory arrest has become the critical factor in the majority of fatalities arising from muscular dystrophy $[16,17]$. While the vast majority of studies evaluating the function of the respiratory muscles have used the diaphragm muscle as model of study, information on the intercostal muscles, in particular at cellular level, is more fragmented [8].

These muscles are also of relevance in Chronic Obstructive Pulmonary Disorder (COPD), given their critical role in manipulating the configuration of the ribs [9] and therefore the overall morphology of the thorax. In addition to the mechanical reorganization of the intercostal muscles that results from the inflated thorax common to COPD patients, clear changes in the expression of myosin heavy chain isoforms have been documented [18].

Here we describe in detail the isolation and preliminary characterization of isolated adherent intercostals muscle fibers in the common laboratory mouse based on techniques and methods previously developed by our laboratory for use on other muscles [19-25]. The loading of fluorescent $\mathrm{Ca}^{2+}$ indicators combined with electrophysiological approaches, including electrical field stimulation of the cultured muscle fibers using any stimulation pattern desired, and the transfection and overexpression of fluorescent fusion proteins permit the study of spatiotemporal aspects of excitation-contraction coupling and biological processes such as excitation-transcription coupling. Due to the difficulty encountered in achieving consistent adhesion of the intercostal fibers to commercial glass-bottom culture dishes, we also include detailed methods for the construction and preparation of glass-bottom dishes optimized for such muscle fiber studies. These dishes have improved the reliability and yield of attached fibers in our preparations several fold. The methods presented here can be modified to allow culture of intercostal fibers from animal at various ages or from animal models with specific genetic background. The protocol described here is intended more generally to provide a flexible new primary cell culture of the as yet poorly characterized, but clinically relevant muscular group, the intercostals.

\section{Methods}

2.1. Animal Use. All animals were euthanized by $\mathrm{CO}_{2}$ exposure followed by cervical dislocation before removal of the muscles according to protocols approved by the University of Maryland Institutional Animal Care and Use Committee.

2.2. Dish Construction. Dishes are constructed from plastic Petri dishes with glass coverslips and sealed using parafilm. Wells are drilled with an $11 / 32^{\prime \prime}$ bit, cleaned with a razor, and washed with $70 \%$ ethanol (Supplemental Figures 1(a)-1(e) available online at doi:10.1155/2011/393740). Multiple wells may be cut per dish to increase plating area (Supplemental
Figure 1(f)); drilling larger holes is not recommended as we have observed that small wells improve adhesion of the fibers.

Parafilm folded to double thickness is cut using the coverslip as a template (Supplemental Figure 2). An unused pencil eraser is useful to hold the coverslip in place, but should be covered with a piece of scotch tape to minimize transfer of oils to the glass. Coverglass is then heated lightly on a hotplate with the parafilm facing up until the parafilm becomes transparent but not liquid. Dishes are pressed firmly over coverslips to seal each well and then parafilm is allowed to cool (Supplemental Figures 2(e)-2(g)). Parafilm from the center of the well is cut out with a scalpel and removed. The seal should be tested with water or media prior to use.

Prior to use, dishes are sterilized by UV irradiation and spotted with approximately $6 \mu \mathrm{L}$ of cold mouse laminin $(1 \mathrm{mg} / \mathrm{mL})$. Laminin is immediately covered with $100 \mu \mathrm{L}$ MEM and the bottom of each well is lightly scratched with the pipette tip to ensure full coverage (Supplemental Figures 2(k) and 2(l)). After at least 1 hour incubation, liquid is removed from the wells and dishes are rinsed twice with MEM and covered with $1 \mathrm{~mL}$ MEM plus $100 \mu \mathrm{L}$ MEM per well.

2.3. Dissection. Following cervical dislocation (see animal use), $70 \%$ ethanol is used to clean the mouse and dissection is carried out in a sterile laminar flow hood to prevent contamination of the culture. Hindfeet are pinned at maximal posterior extension, and forepaws are pinned at maximum lateral extension with the ventral face of the mouse up (Figure $1(b))$. Skin is cut, posterior to anterior, from the lower abdomen to the throat (Figure 1(c)), and a lateral incision is made with a scalpel at the posterior end of the cut to pin back the skin (Figure 1(d)). Skin is removed by gently raking the scalpel against the interface between skin and subcutaneous tissue (Figures 1(e) and 1(f)) until the vertebral column is nearly exposed. An incision is made at the anterior end of the abdominal cavity, exposing the liver (Figure $1(\mathrm{~g})$ ). When the liver is pulled back, the diaphragm is exposed, although difficult to visualize. The thoracic cavity may be punctured by following the dorsal face of the sternum with a scalpel, causing the thoracic cavity to inflate (Figure $1(\mathrm{~h})$ ). This makes the diaphragm easier to see and remove by cutting along the posterior internal edge of the rib cage (Figure 1(i)). Once the diaphragm is free, an incision is made along the ventral face of the sternum and the superficial muscles of the thorax are removed in a similar fashion to the skin (Figures $1(j)-1(1))$. This exposes the intercostal muscles which are then immediately bathed in MEM to prevent drying. One half of the rib cage is then excised by using scissors to cut along the sternum (Figure $1(\mathrm{~m})$ ) and thoracic vertebrae (Figure $1(n))$. The half rib cage is then placed in MEM to rinse away excess debris (Figure $1(\mathrm{o})$ ).

2.4. Digestion and Fiber Isolation. The media used to rinse the ribs is removed and replaced with MEM containing $2 \mathrm{mg} / \mathrm{mL}$ collagenase and $5 \mathrm{mg} / \mathrm{mL}$ dispase which has been passed through a $0.2 \mu \mathrm{m}$ filter. The ribs are then incubated at $37^{\circ} \mathrm{C}$ and $5 \% \mathrm{CO}_{2}$ for 3 hours (older mice may require 


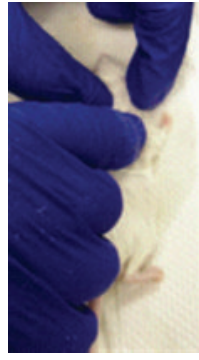

(a)

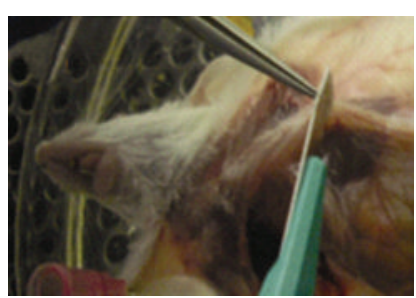

(g)

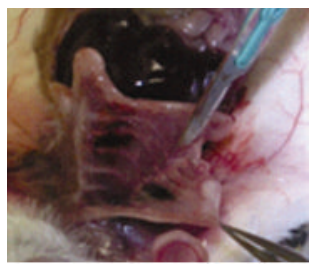

(k)

(b)
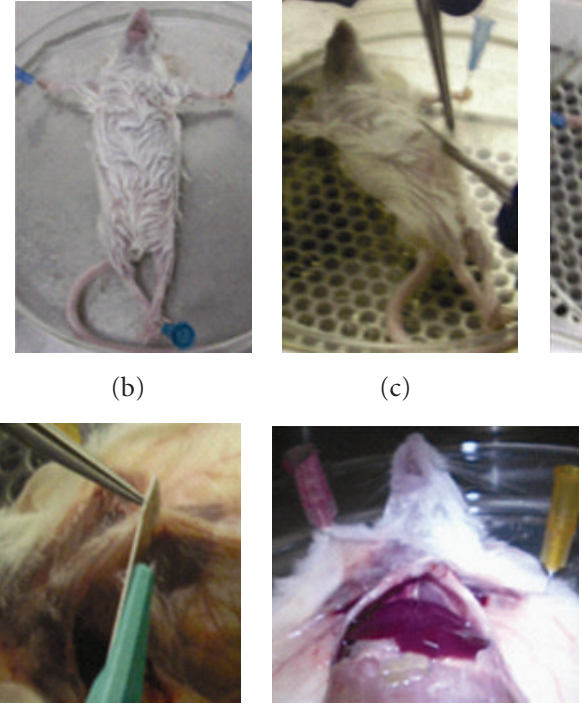

(h)

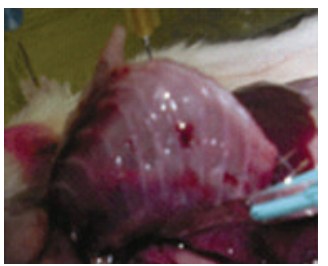

(1)

(c)
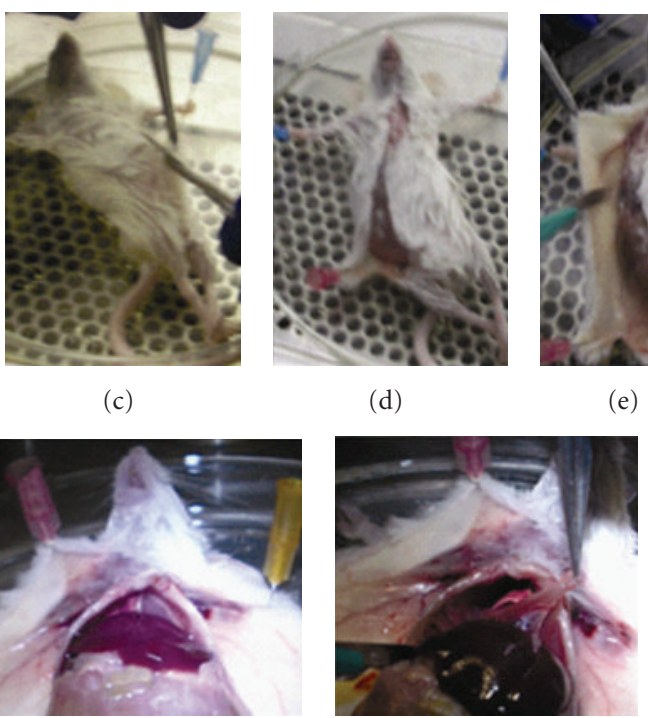

(i)

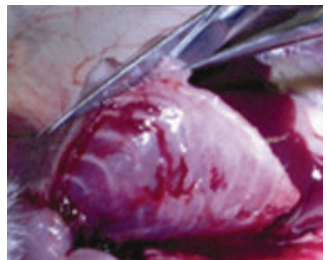

(m)

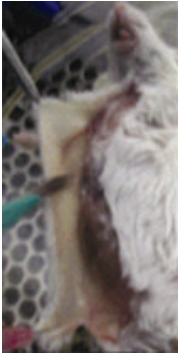

(e)

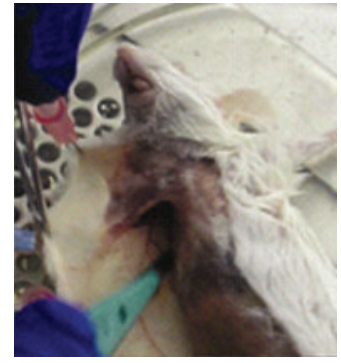

(f)

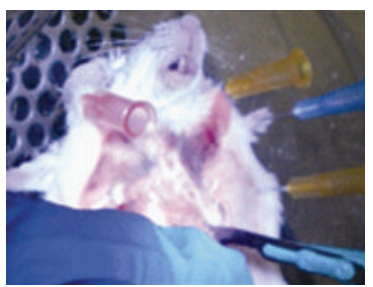

(j)

FIgure 1: Critical Steps in Gross Dissection of Intercostal Muscles. (a) Cervical dislocation following $\mathrm{CO}_{2}$ asphyxiation and $70 \%$ ethanol wash. (b) Pinning mouse, ventral face up. (c) First incision from posterior abdomen to throat. (d) Skinning: pinning back initial flap. (e) Skinning: cutting subcutaneous connective tissue. (f) Skinning: continue removal to vertebral column. (g) Opening incision at anterior end of abdominal cavity. (h) Exposed posterior face of thoracic cavity (after puncture). (i) Removal of diaphragm. (j) Incision along ventral face of sternum. (k) Removal of pectoral and other muscle groups. (l) Full exposure of ribs. (m) Severing ribs (ventral face). (n) Severing ribs (dorsal face). (o) Isolated ribs transferred to HBSS for imaging. Scale is centimeter ruler.

longer incubation). Following digestion, the ribs are transferred into MEM supplemented with 10\% FBS and the procedure is continued under a dissection microscope. Excess vasculature and fragments of nonintercostal muscle fragments are removed with forceps. A single band of intercostal muscle is isolated with its flanking ribs (Figure 2(a), white arrows) by pulling the flanking ribs away from the next nearest (Figure 2(a), black arrows).

The isolated rib pair and the intercostal band are transferred to a clean $60 \mathrm{~mm}$ dish containing $7 \mathrm{~mL}$ of MEM supplemented with 10\% FBS (Figure 2(b)). Fibers are triturated using a large bore flame polished pipette (Figure 2(c), Supplemental Figure 3) until a significant population of isolated fibers is obtained (Figure 2(d)). If muscle tissue detaches completely from ribs, a smaller bore pipette may be used. The dish is gently swirled to aggregate fibers (Figure $2(\mathrm{e})$ ). If large amounts of debris are floating on the surface of the media, it may be removed. A wide bore micropipette tip is used to gather $8-12$ healthy fibers per well from the periphery of the fiber cluster. Fibers are plated directly onto the glass surface in each well of the dishes prepared earlier. Fibers may be damaged or stick to the interior of the tip. These are removed and replaced; however, overplating can cause fibers to mutually interfere with attachment and is to be avoided. Large pieces of debris or dying fibers are removed with forceps if it is possible to do so without disturbing healthy fibers. After several minutes to allow initial attachment, dishes are transferred to the incubator overnight to complete attachment. We note that fibers often attach near the periphery of the dish even when plated initially in the center.

In all cases, fibers are maintained in media without serum following dissociation. The removal of serum from the media following dissociation of the fibers mitigates gross morphological changes, proliferation of secondary cells, and some changes in calcium signaling $[26,27]$.

2.5. Digestion Conditions of Nonintercostal Fibers. Dispase is omitted from the digestion of both soleus and FDB muscles. FDBs (but not soleus) may be fully digested in as little as 2.5 hours, particularly if the mouse used is young. Apart from removal of bone (not required for either soleus or FDB), plating may proceed exactly as with the intercostal fibers, although a smaller bore pipette may be used for trituration if desired. 


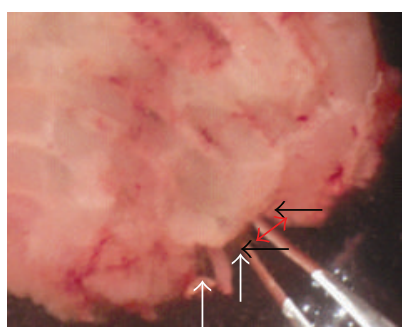

(a)

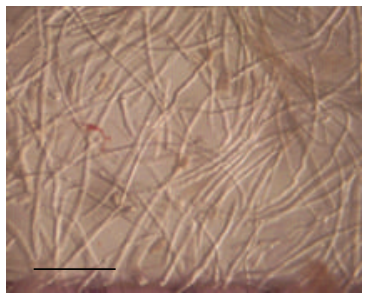

(e)

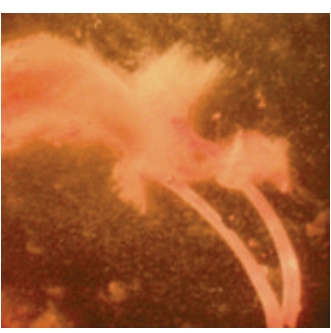

(b)

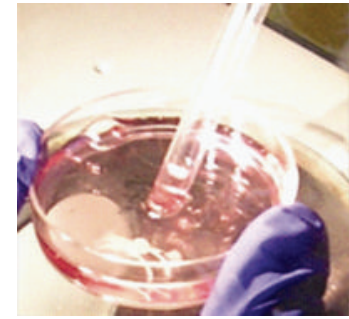

(c)

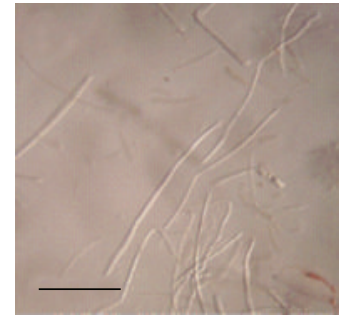

(d)

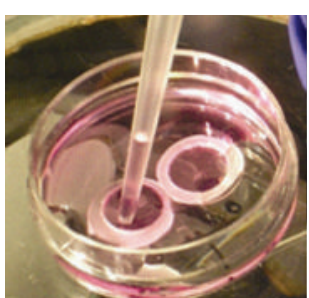

(f)

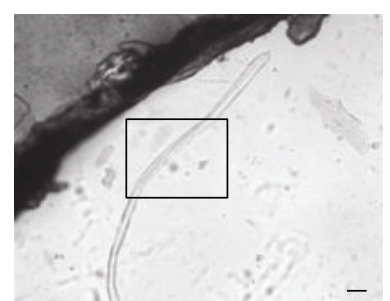

(g)

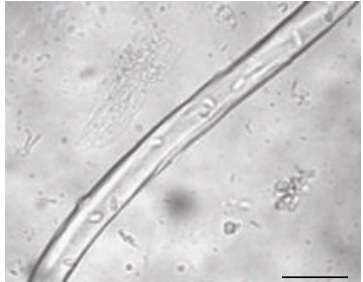

(h)

Figure 2: Dissociation. Following digestion, ribs are transferred into MEM containing 10\% FBS and gentamycin (a). Rib pair flanking the desired muscle (white arrows) is isolated (b) and transferred into clean $60 \mathrm{~mm}$ Falcon Petri dish containing $7 \mathrm{~mL}$ MEM with $10 \%$ FBS and gentamycin. Fibers are triturated (c) until a significant population of fibers are isolated (d). Fibers may be aggregated in the center of dish (e) by shaking gently in a circular motion. FinnTip 250 Wide pipette tips are used to plate fibers (f) to prevent damage from fluidic shearing. Brightfield images of an adhered fiber at low (g) and high (h) magnification. Scale bars are approximately $1 \mathrm{~mm}(\mathrm{~d}, \mathrm{e})$ or $50 \mu \mathrm{m}(\mathrm{g}, \mathrm{h})$.

2.6. Indo1 Ratiometric Recordings. Cultured FDB or intercostal fibers were loaded with Indo-1 AM (Invitrogen, Eugene, OR) at a bath concentration of $2 \mu \mathrm{M}$ for $30 \mathrm{~min}$ at $22^{\circ} \mathrm{C}$. Then, the fibers were washed thoroughly with $\mathrm{L}$ 15 media to remove residual Indo-1 AM and incubated at $22^{\circ} \mathrm{C}$ for another $30 \mathrm{~min}$ to allow dye conversion. The culture dish was mounted on an Olympus IX71 inverted microscope and viewed with an Olympus 60x/1.20 NA water immersion objective. Fibers were illuminated at $360 \mathrm{~nm}$, and the fluorescence emitted at 405 and $485 \mathrm{~nm}$ was detected simultaneously. The emission signals were digitized and sampled at $10 \mathrm{~Hz}$ using a built-in AD/DA converter of a EPC10 amplifier and the acquisition software Patchmaster (HEKA, Instruments). Field stimulation (square pulse, $14 \mathrm{~V} \times 1 \mathrm{~ms}$ ) was produced by a custom pulse generator through a pair of platinum electrodes. The electrodes were closely spaced $(0.5 \mathrm{~mm})$ and positioned directly above the center of the objective lens, to achieve semilocal stimulation. Only fibers exhibiting reproducible and consistent responses to field stimulation of alternate polarity were used for the analysis.

2.7. Fluo-4 $\mathrm{AM} \mathrm{Ca}^{2+}$ Recordings. Fluo-4 AM (Invitrogen, Eugene, OR) loading, high speed line scan $x$ - $t$ imaging on a Zeiss LSM 5 Live confocal system, and image analysis were all performed as previously described [25]. Fibers were stimulated using parallel platinum field electrodes, with acquisition of the Fluo4 confocal line scan image synchronized to the field stimulus to generate a temporal profile.

2.8. Exogenous Protein Expression and Imaging. The recombinant adenovirus of Glut4-GFP was kindly provided to our laboratory by Dr. Jeffrey Pessin. The expression plasmid of
NFATc1 cDNA was a gift from Dr. Gerald R. Crabtree. The construction of recombinant adenoviruses of NFATc1-GFP as well as the procedures for infection with recombinant adenoviruses of single muscle fibers has been previously described [24]. Cultures were maintained in MEM in a $37^{\circ} \mathrm{C}$, $5 \% \mathrm{CO}_{2}$ incubator until expression levels were appropriate for imaging, then transferred into Liebovitz L-15 (GIBCO) for microscope work. Images were taken using an Olympus IX70 inverted microscope equipped with an Olympus Fluoview 500 laser scanning confocal imaging system using an Olympus 60x/1.2 NA water immersion objective. A $488 \mathrm{~nm}$ excitation wavelength and $505 \mathrm{~nm}$ long-pass emission filter were used to visualize GFP.

2.9. Data Analysis. Line-scan Fluo4 images were processed using LSM examiner (Carl Zeiss, Jena). GFP and Glut4GFP confocal images were processed using ImageJ (NIH, Bethesda, MD, USA; http://rsb.info.nih.gov/ij/). Indol and fluo4 $\mathrm{Ca}^{2+}$ signals and statistical analysis were conducted using Origin pro 8 (OriginLab Corporation, Northampton, MA, USA). Summary data were reported as mean \pm SEM. Statistical significance was assessed using parametric unpaired two sample $t$-test. Differences were considered significant when $P$ value $<0.05$.

Indo1 data was reported as the ratio of fluorescence emission at $405 \mathrm{~nm}$ to emission at $485 \mathrm{~nm}$ (indol ratio) which correspond to the peak values of the calcium bound and unbound forms, respectively, or the difference in indo 1 ratio between peak and resting values ( $\Delta$ indol ratio). Fluo4 data was reported as the difference between fluorescence intensity and an average value of fluorescence taken when the fiber was at rest, normalized to the resting value $\left(\Delta F / F_{0}\right)$. 


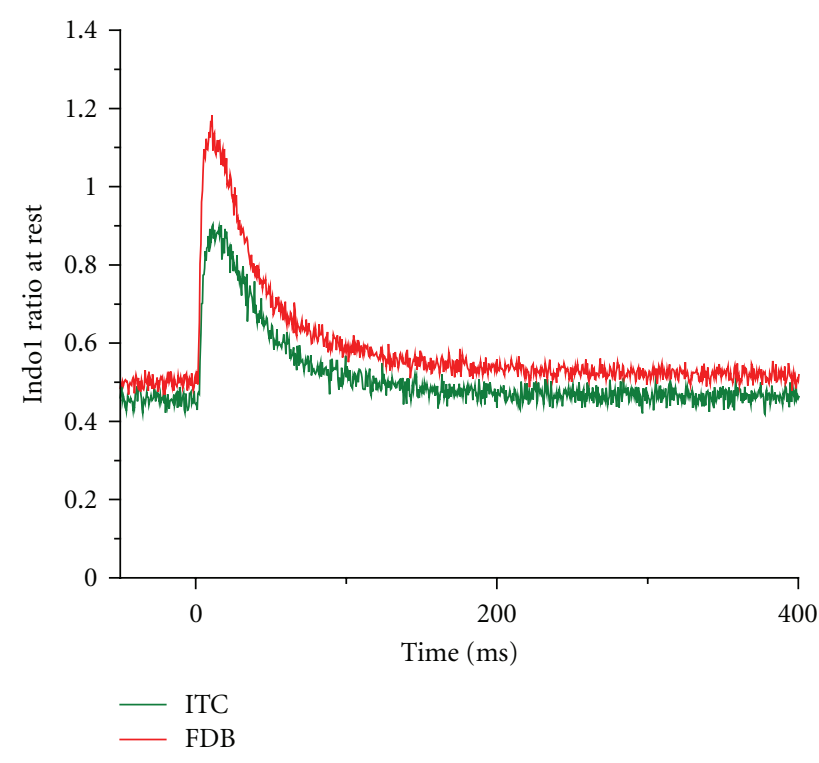

(a)

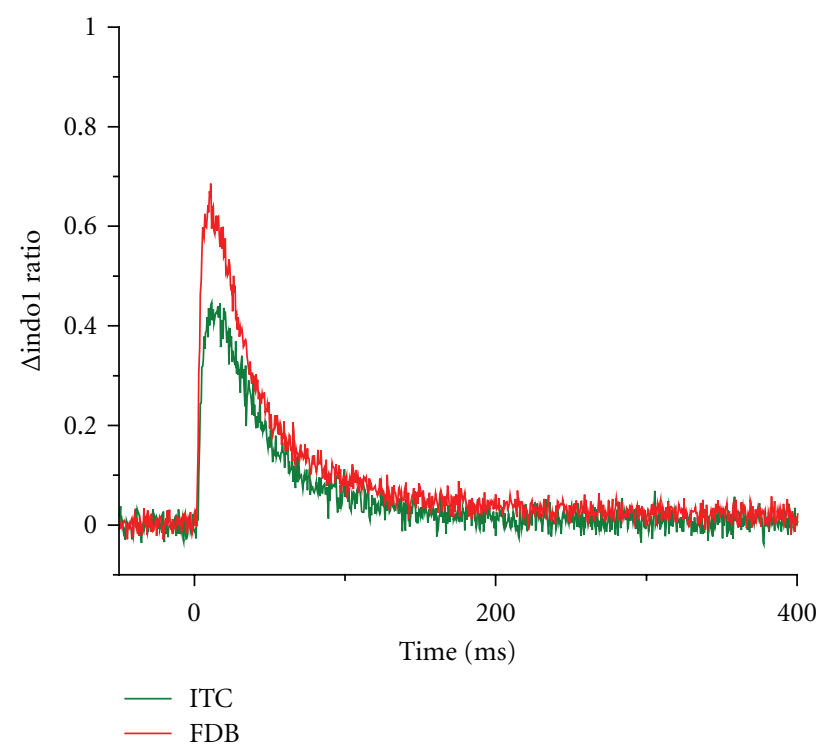

(c)

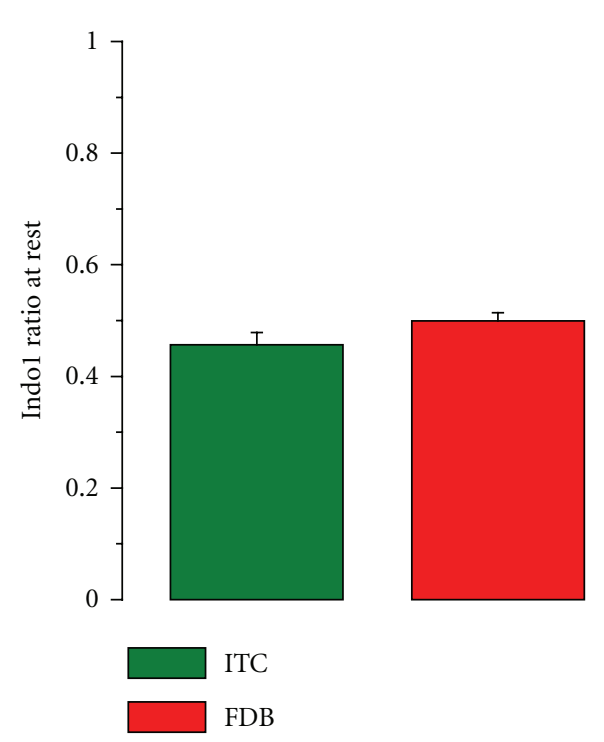

(b)

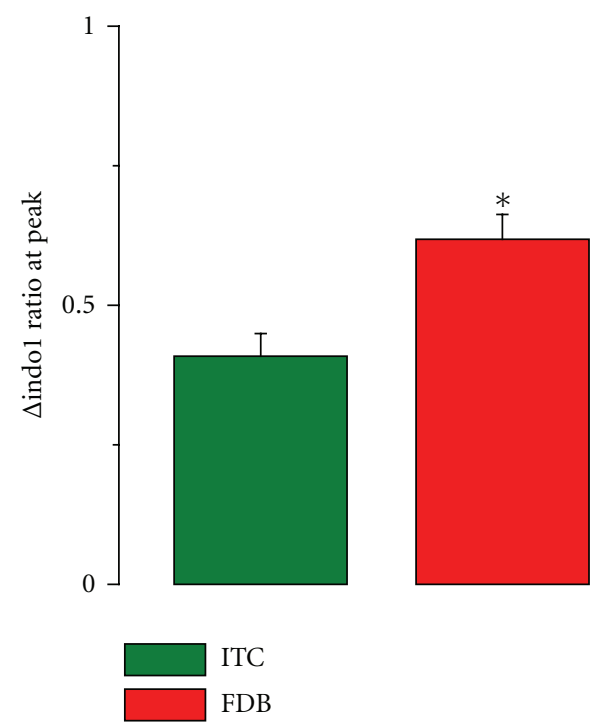

(d)

FIGURE 3: Intercostal fibers exhibit decreased peak amplitude of the Indo- $1 \mathrm{Ca}^{2+}$ transient following a single action potential. (a) Average Indo-1 ratio $\mathrm{Ca}^{2+}$ transients from FDB (red trace; $n=9$ ) and Intercostal (ITC; green trace; $n=11$ ) fibers. Isolated fibers were stimulated with field electrodes at time zero and emission ratio was examined. (b) Bar plot summarizing resting Indo-1 ratio averages of FDB and ITC fibers. No significant differences in resting indol ratio were detected between groups $(P=0.12)$. (c) Traces from panel (a), expressed as $\Delta$ indo-1 ratio (Indo1 ratio-Indol ratio at rest). (d) Bar plot representation of peak $\Delta$ Indo-1 ratio shown in $(c), F D B=0.61 \pm 0.04, n=9$; ITC $=0.40 \pm 0.04, n=11 ;{ }^{*} P=0.0015$. Error bars in (d) are the SE values. ITC fibers displayed significantly reduced Ca ${ }^{2+}$ transients when compared with FDB fibers.

Data from the NFATc1-GFP fusion protein was reported as the ratio of fluorescence intensity in the nucleus to the intensity in the cytoplasm $(\mathrm{N} / \mathrm{C})$ in order to correct for variations in expression level between fibers. Raw data for both Fluo4 and NFATc1 was corrected for background before analysis by subtracting the intensity measured in a region outside the muscle fiber.

\section{Results}

3.1. Gross Morphology. The intercostal muscles are composed of two overlapping sheets (inner and outer) of muscle fibers with roughly perpendicular orientations. Each muscle spans diagonally between two ribs from the vertebral column to the sternum. Following dissection connective tissues between 


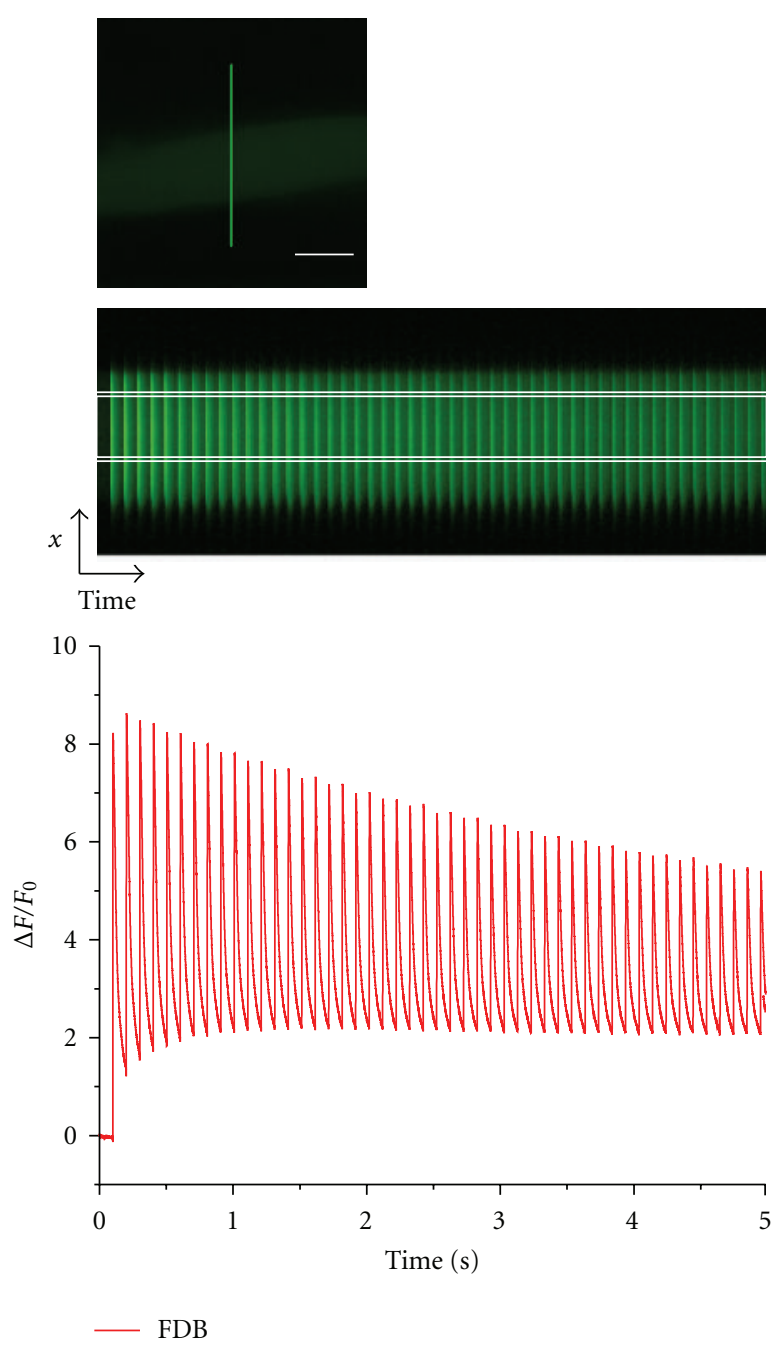

(a)

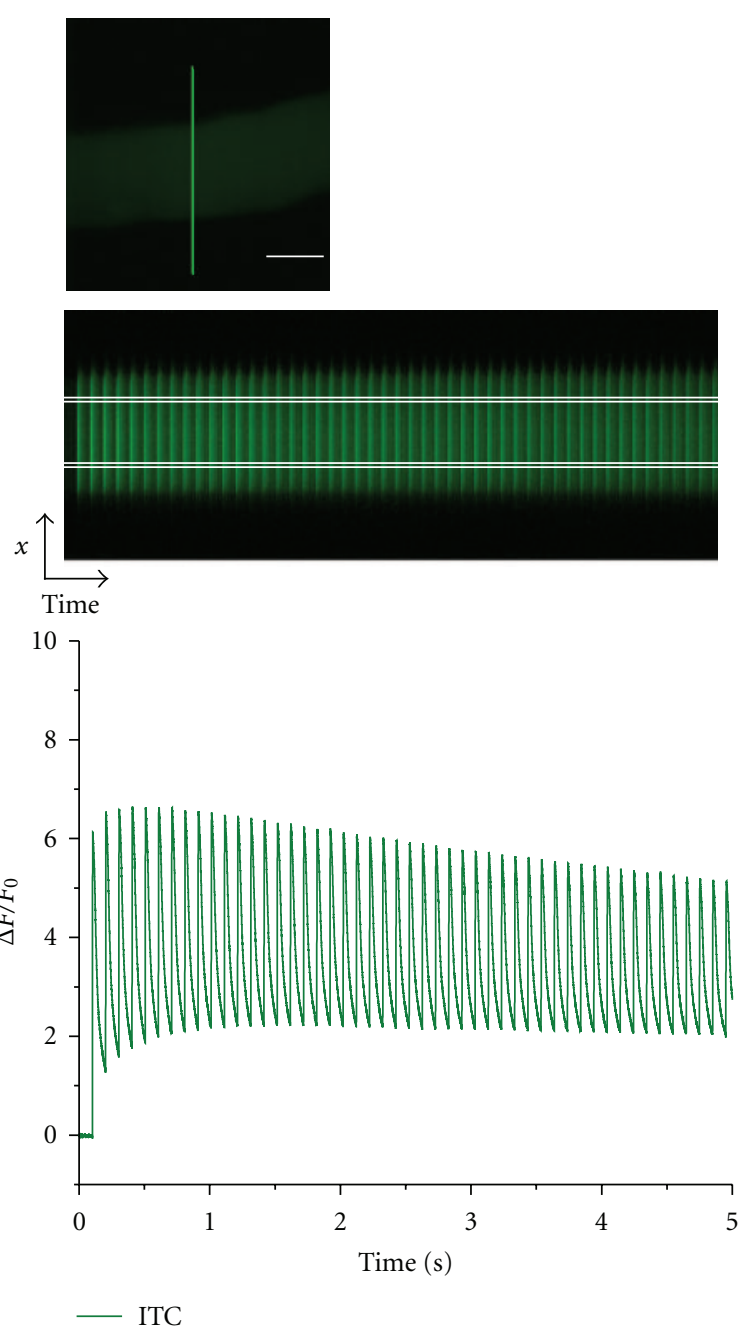

(b)

FIGURE 4: Fluo4 $\mathrm{Ca}^{2+}$ transients elicited by field stimulation in intercostal and FDB fibers. Representative line-scan images of $\mathrm{Ca}^{2+}$ transient responses in FDB (a) and intercostal (b) fibers to a $5 \mathrm{sec} 10 \mathrm{~Hz}$ train of field stimulation applied at time zero, performed with nonratiometric fluo-4 AM loaded fibers and assayed with high-speed confocal line scan microscopy (100 $\mu \mathrm{s} /$ line). Images in top of panels (a) and (b) show $x y$ images of FDB and intercostals fibers to indicate the location of the scan line, scale bar is $20 \mu \mathrm{m}$. Note that fluo-4 signal at rest in these fibers is very low. Traces under the images are the time courses of the fluo $4 \mathrm{Ca}^{2+}$ transients. The white lines in the time domain images indicate the region of interest drawn at the center of the fiber and used to calculate the change in fluorescence in response to field stimulation. Data presented as $\Delta F / F_{0}$. Fluorescence was detected with a Zeiss LSM 5 Live ultrafast confocal system based on an Axiovert 200M inverted microscope. Fibers were imaged with a $63 \mathrm{x} / \mathrm{NA} 1.2$ water immersion objective lens. Excitation for fluo- 4 was provided by the $488 \mathrm{~nm}$ line of a $100 \mathrm{~mW}$ diode laser, and emitted light was collected at $>510 \mathrm{~nm}$.

muscle and ribs are not readily appreciable at the macroscopic scale (Figure 1).

3.2. Isolating Intercostal Fibers. The initial attempt to isolate and culture intercostal muscle fibers was based on our existing protocols for isolating and culturing mouse Flexor Digitorum Brevis (FDB) fibers [21-25], which are maintained in commercially available glass-bottomed dishes. After an initial development and optimization of dissection and enzymatic treatment methods, we achieved a high fraction of viable isolated intercostal fibers. These fibers maintained clear striations and twitch response for several days, but did not reliably form adherent cultures, even after extensive experimentation with a variety of substrates derived from extracellular matrix. During the course of unrelated work, we noted that customized glass-bottomed dishes which we had designed with smaller diameter wells using a different glass substrate seemed to facilitate attachment in our FDB model (see Supplemental Figures 1 and 2). Plating intercostal fibers in these dishes dramatically improved the attachment rate and reliability of the culture.

After fiber isolation, our procedure yields a heterogeneous mix of internal and external intercostal fibers with a successful attachment rate around 50\%. Isolated intercostal fibers (Figure 2) are similar to FDBs in width, but 4-5 times the length. This additional length makes the fibers 


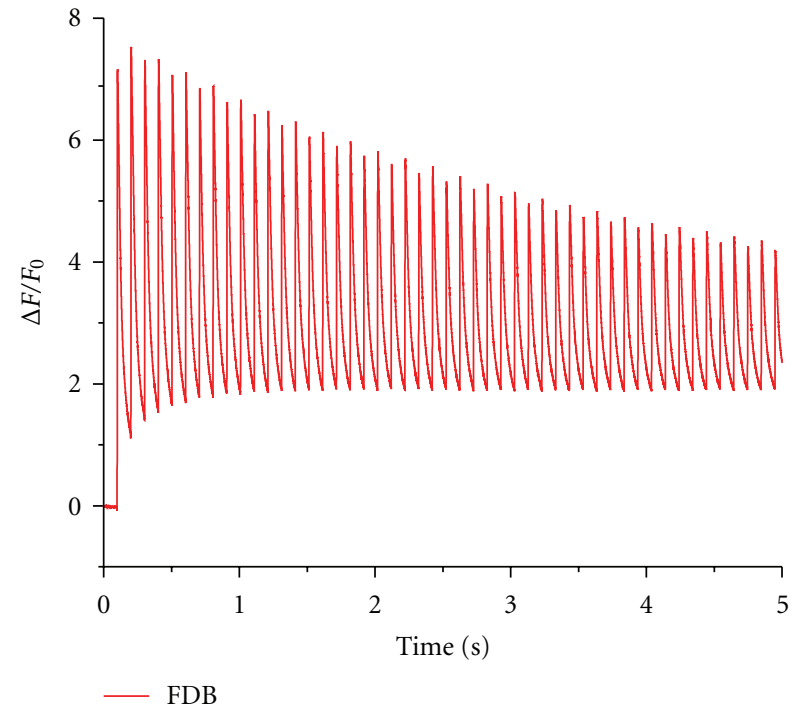

(a)

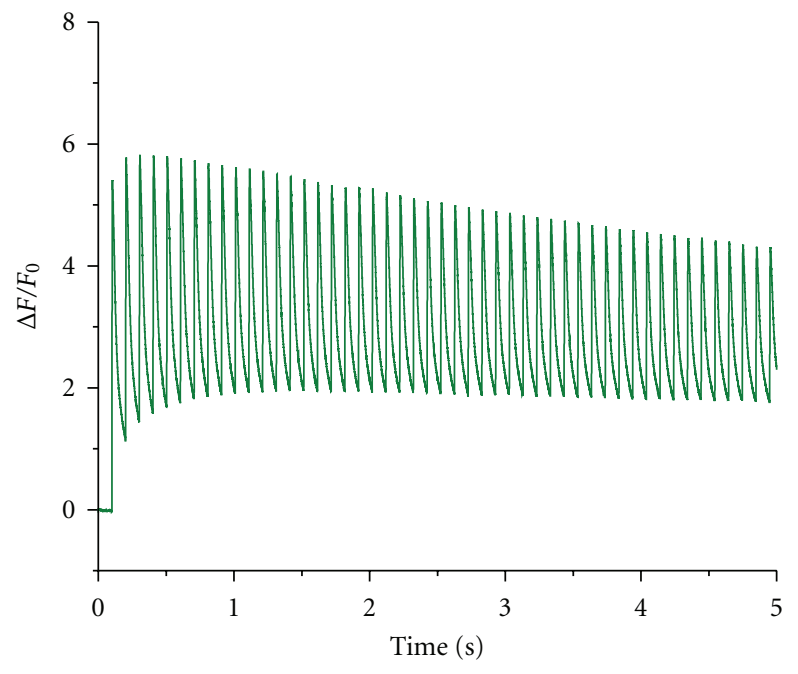

- ITC

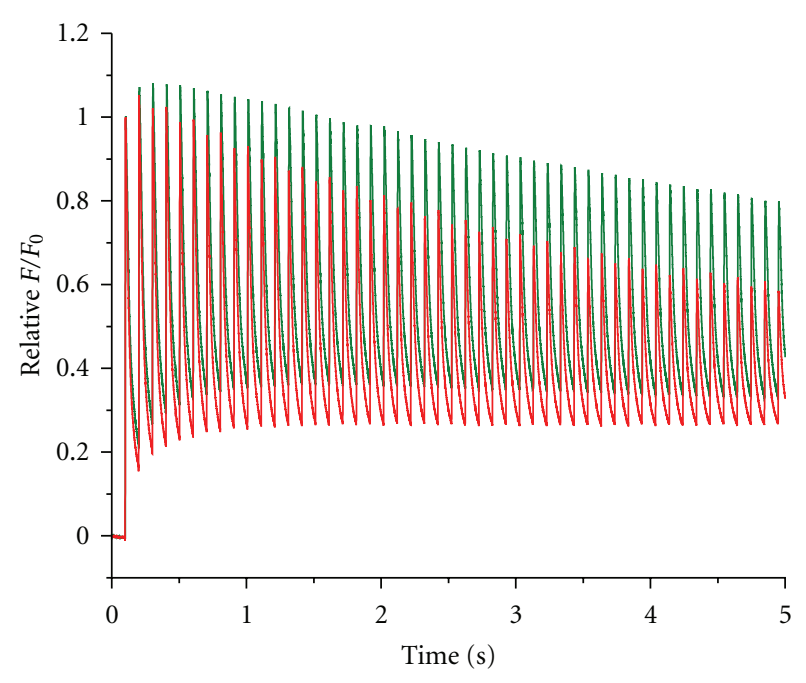

(b)

(c)

FIgURE 5: Comparison of electrically evoked $\mathrm{Ca}^{2+}$ transients between intercostal and FDB fibers. Average fluo4 $\mathrm{Ca}^{2+}$ transient from FDB $((\mathrm{a})$, red trace; $n=9)$ or intercostals fibers ((b), ITC, green trace; $n=9)$ in response to $5 \mathrm{sec} 10 \mathrm{~Hz}$ train field stimulation. Traces in (c) were normalized to the amplitude of the initial response. At the end of the $5 \mathrm{sec}$ train, ITC fibers demonstrated significantly smaller relative decrease of $\mathrm{Ca}^{2+}$ transient amplitude compared to FDB counterparts $(P<0.05)$. ITC muscle fibers exhibit decreased peak amplitude of the fluo- $4 \mathrm{Ca}^{2+}$ transients in response to a single field stimulus; however, the intercostals are able to maintain consistent levels of calcium during repetitive stimulation. Fluorescence was detected as in Figure 4.

susceptible to bends when they are fully attached (see Figure $1(\mathrm{~g}))$. Almost all of the whole fibers that attach to the glass display clear striations (Figure 6) and respond to external electrical field stimulation administered either as single pulses or repetitive trains with twitching similar to that of FDBs (see supplemental videos 1 and 2).

In order to examine the general applicability of these dishes, we also used them to plate fibers from the slow-type soleus muscle, which also displays poor adherence characteristics [22]. Although we used a less stringent digestion procedure, plating was carried out exactly as for intercostals.
The exceptional length of the soleus fibers causes them to interfere with each other and permits the formation of many bends and kinks which prevent complete attachment. Nonetheless, the fibers were responsive to electrical stimulation and adhered securely enough to withstand repeated twitching (see supplemental video 3) more than 24 hours after plating. Successful attachment rate was variable between the wells plated, but overall comparable to the intercostals.

\section{3. $\mathrm{Ca}^{2+}$ Homeostasis and Electrically Evoked $\mathrm{Ca}^{2+}$ Transients in Intercostal Fibers. Primary cultured intercostal fibers}




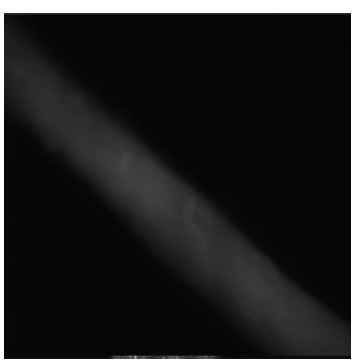

(a)

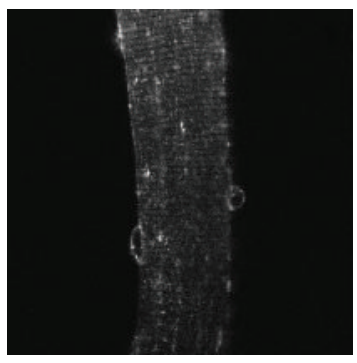

(e)

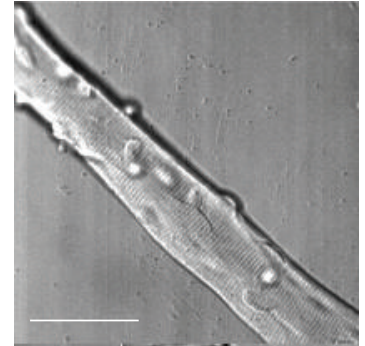

(b)

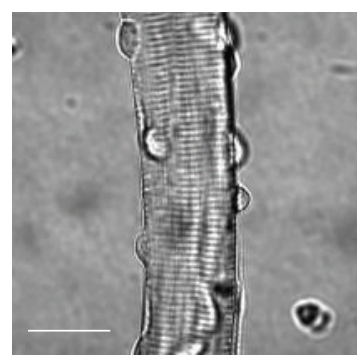

(f)

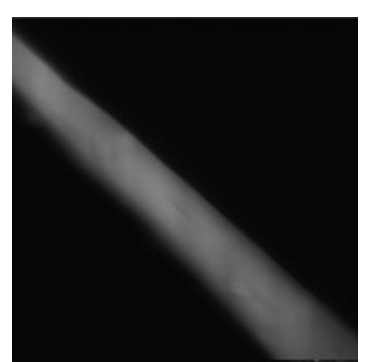

(c)

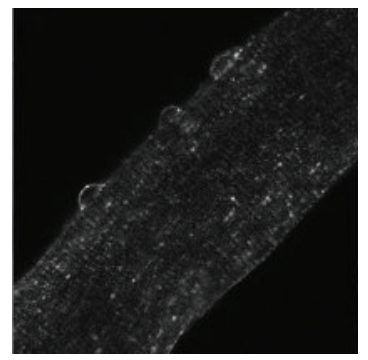

(g)

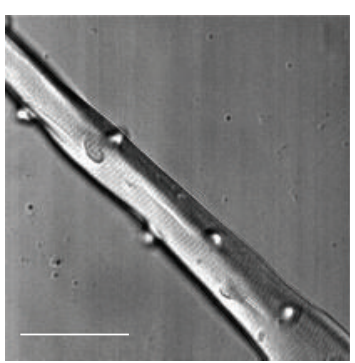

(d)

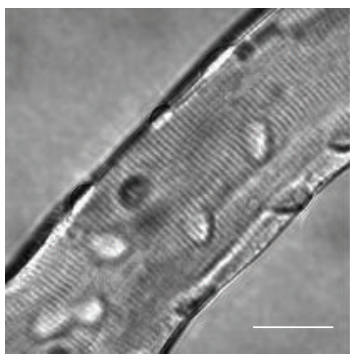

(h)

FIGURE 6: Overexpression of foreign proteins in intercostal fibers in culture. FDB (a, b, e, f) and intercostal (c, d, g, h) muscle fibers expressing either GFP (a-d) or a Glut4-GFP fusion construct (e-h). Scale bars are $50 \mu \mathrm{m}(\mathrm{b}, \mathrm{d})$ or $25 \mu \mathrm{m}(\mathrm{f}, \mathrm{h})$. Images were acquired as in Figure 4 for panels (a-d) or with an Olympus FluoView 500 confocal system, built on an Olympus IX71 inverted microscope for panels (e-h). Fibers were imaged viewed with a UPlan Apo 60x/NA 1.20 water-immersion objective lens using $488 \mathrm{~nm}$ excitation (Multiline Argon laser) for GFP with emission detected with $505 \mathrm{~nm}$ long-pass filter. Photobleaching and photodamage were reduced by operating the lasers at the lowest possible power that still provided images with satisfactory signal to noise ratio. Brightfield images in panels (b, d, f, h) were adjusted for improved contrast using ImageJ.

retain many properties of native fibers, including contractions and $\mathrm{Ca}^{2+}$ transients in response to electrical field stimuli (Figures 3, 4, and 5; see Supplemental Video 1). A brief comparative study between intercostal fibers and the established FDB isolated fiber model was also conducted (Figures 3, 4, and 5). First, we sought to evaluate $\mathrm{Ca}^{2+}$ handling properties in single, intact indo1-loaded intercostal $(n=8)$ and FDB $(n=16)$ muscle fibers stimulated by an action potential (AP) elicited by a $1 \mathrm{~ms}$ electric field stimulus (Figure 3). While resting indol ratio prior to stimulation was not significantly different between intercostal $(0.45 \pm 0.02$; $n=11)$ and FDB fibers $(0.49 \pm 0.01$; Figures $3(\mathrm{a})$ and $3(\mathrm{~b})$; $n=9, P=0.12)$, the peak of the indol $\mathrm{Ca}^{2+}$ transient following a single AP ( $\triangle$ indol ratio) was significantly smaller in intercostal fibers than in the FDB fibers (intercostal $=0.40$ $\pm 0.04, \mathrm{FDB}=0.61 \pm 0.04, P=0.0015$, Figures $3(\mathrm{c})$ and $3(d))$. These results suggest that intercostal fibers exhibit a decreased AP-evoked $\mathrm{Ca}^{2+}$ release.

To allow better temporal resolution of the $\mathrm{Ca}^{2+}$ transients, we next monitored fluo- 4 transients during repetitive stimulation of intercostal and FDB fibers using an ultra highspeed ( $100 \mu$ s/line) confocal microscope in line scan mode. Figure 4 illustrates representative $x$ - $t$ confocal images and corresponding fluo4 $\mathrm{Ca}^{2+}$ transients, expressed as $\Delta F / F_{0}$, from an FDB (Figure 4(a)) and an intercostal (Figure 4(b)) fiber during a 5-second $10 \mathrm{~Hz}$ train. Line-scan fluorescence images were obtained along a line perpendicular to the long axis of the fiber (Figures 4(a) and 4(b), top panels). Successive vertical lines in each image reveal the time course (left to right; middle panels) at $100 \mu$ s resolution of the fluorescence signal along the scanned line before and during the repetitive stimulation. Bottom panels in Figures 4(a) and 4(b) show the time course of the fluo4 fluorescence. Figure 5 shows average responses from FDB (red trace; Figure 5(a)) and intercostal (green trace; Figure 5(b)) fibers stimulated by a 5 -second $10 \mathrm{~Hz}$ train. Consistent with the Indo-1 data (Figure 3), fluo4 $\mathrm{Ca}^{2+}$ transients appear suppressed in intercostal fibers (Figure 5(b)) in comparison to those observed in FDB fibers (Figure 5(a)).

However, the intercostals are able to maintain a more consistent level of calcium release during repetitive stimulation (Figure 5(b)). By normalizing the traces elicited by the $10 \mathrm{~Hz}$ trains to the amplitude of the first fluo4 transient in their respective train, the relative differences in $\mathrm{Ca}^{2+}$ transient summation as well as the properties of decaying phase during the train can be appreciated (Figure 5(c)). Both intercostal and FDB fibers showed an initial increase in the peak amplitude during the $10 \mathrm{~Hz}$ train protocol, exceeding the peak value of the initial stimulation until 2 and 0.5 seconds, respectively (Figure 5(c)). Additionally, the relative amplitude of the $\mathrm{Ca}^{2+}$ transients during the $10 \mathrm{~Hz}$ train declines more slowly in intercostal fibers (Figure 5(c)).

Unexpectedly, subpopulations reflecting an anticipated difference between fiber types were not observed in the intercostal fibers. However, we suspect that this is not due to genuine homogeneity, but rather a limitation of the calcium ion equilibration kinetics of fluo-4 in distinguishing such sub-populations, which may be quite similar. 


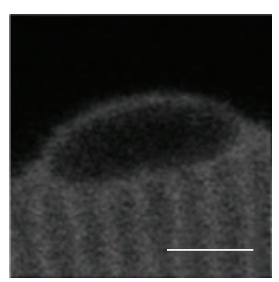

(a)

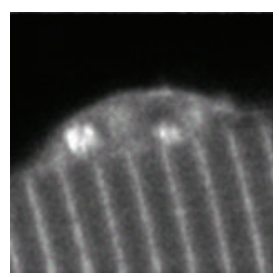

(d)

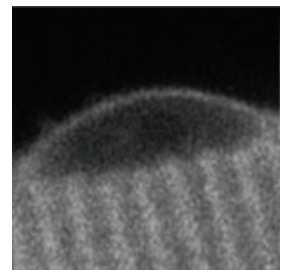

(b)

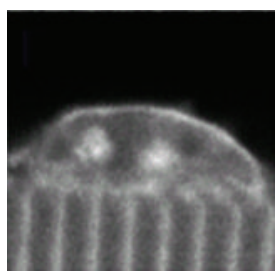

(e)

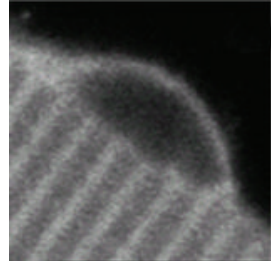

(c)

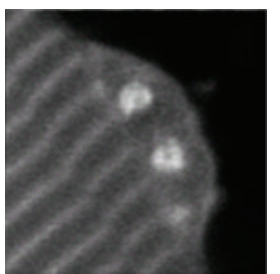

(f)

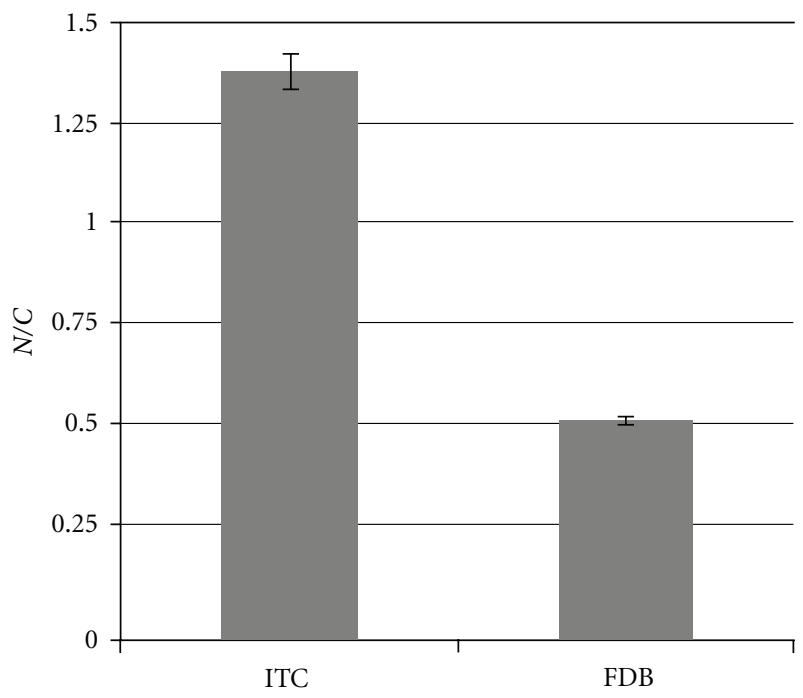

(g)

Figure 7: NFATc1-GFP distribution in intercostal and FDB fibers. FDB (a-c) and intercostal (d-f) fibers expressing NFATc1-GFP imaged at rest after 3 days in culture. Scale bar, $5 \mu \mathrm{m}$. Nuclei in intercostal fibers exhibit significantly elevated levels of NFATc1 when compared to FDBs cultured under the same conditions $(\mathrm{g}), N / C$ ratio of NFATcl-GFP in ITC $=1.38 \pm 0.05, n=102$ nuclei versus $0.51 \pm 0.01, n=42$ nuclei, in FDB fibers, $P<0.05)$. Fluorescence was detected as in Figure 6.

These results lead us to conclude that adherent cultures of intercostal fibers are a new and unique model for the study of $\mathrm{Ca}^{2+}$-signaling pathways at the cellular and subcellular levels by using ultrafast confocal imaging combined with $\mathrm{Ca}^{2+}$ sensitive fluorescent probes.

3.4. Expression of Exogenous Proteins. In order to assess the viability of the intercostal cultures for imaging studies utilizing fluorescent proteins, fibers in culture were exposed to crude lysates of recombinant adenoviral vectors expressing GFP. Exposure to virus was carried out overnight, beginning immediately after plating. Vector dosage was calibrated by expression levels in similarly treated FDB fibers. Cultures were maintained in an incubator to allow expression prior to imaging. Expression of GFP was robust after 48 hours and did not appear to damage the fibers, which maintained clear striations (Figures 6(b) and 6(d)).
We also exposed other cultures to a viral vector expressing a Glut4-GFP fusion protein. Expression continued through 96 hours (Figure 6(g)). Glut4-GFP was expressed in intercostal fibers in puncta both around the nucleus and in lines parallel to the axis of the fiber (Figures 6(e) and 6(g)). This distribution is similar to what we observe in FDB fibers infected with the same construct, as well as the distribution of the native transporter characterized by immunoflu orescence [28], although we observe weaker striations, presumably due to lack of exposure to insulin during the culture period reducing the Glut4 present in the transverse tubule system.

Finally, we exposed the fibers to a viral construct expressing a NFATc1-GFP fusion construct. NFATcl is a transcription factor canonically regulated by the calciumdependent phosphatase calcineurin [29, 30]. Typically resting FDB fibers exhibit low translocation of NFATc1 into 
the nucleus (Figures 7(a), 7(b), and 7(c)) [24]. Unexpectedly, ITC fibers exhibited a higher nuclear distribution of NFATc1GFP at rest (Figures 7(d), 7(e), and 7(f)) when compared to FDB fibers (Figures $7(\mathrm{a}), 7(\mathrm{~b})$, and $7(\mathrm{c})$ ). The resting nuclear/cytosolic $(N / C)$ ratio of NFATc1 was significantly elevated in intercostal fibers (Figure $7(\mathrm{~g})$ ) compared to FDB $(1.38 \pm 0.05, n=102$ nuclei versus $0.51 \pm 0.01, n=42$ nuclei, resp.; $P<0.05)$.

\section{Discussion}

Adult skeletal muscle fiber culture currently focuses on only a handful of the myriad available muscles in the body, extrapolating from the few established culture systems like the FDB to the body as a whole. While it is generally true that various skeletal muscles resemble one another, there is significant divergence among them with respect to their physiology and the expression levels and isoforms of many muscle specific and metabolic proteins $[1,5-7]$. This is dramatically illustrated by the high specificity of pathology in many skeletal muscle disease states resulting from defects in specific genes (e.g., the disproportionate targeting of facial, shoulder, and limb muscles in facioscapulohumeral muscular dystrophy or the extremities in distal muscular dystrophy [17]).

The intercostal fiber cultures introduced here themselves appear to be nearly as versatile as the more established FDB cultures. While we have not yet used intercostal cultures in applications requiring large numbers of adherent fibers, they have proven viable for a wide variety of single fiber experiments, even after several days in culture. Previous work related to the physiology of intercostal muscles comes from studies utilizing organ culture of intact muscle fiber bundles [31-35]. Whereas intercostal muscle fiber bundles offer the advantage of preserving nerve-muscle interactions, as with many organ culture systems it may not be well suited for single cell electrophysiology and high spatial resolution imaging. Furthermore, the ability to mimic stimulation patterns that may be experienced by the muscle during stressed states in vivo (such as hyperventilation or coughing) at the single muscle fiber level offers the opportunity to isolate the response of the muscle from that of the surrounding tissue in a manner not possible with organ culture methods. A variant of the single intercostal fiber isolation was described previously and was used to study satellite cell differentiation cocultured with nonadherent intercostal fibers derived from goats [36]. While these methods are valuable, the availability of adherent single fiber models for imaging and electrophysiology substantially expands our ability to study these muscle types.

The value of studying each skeletal muscle as a separate tissue is demonstrated by our unexpected observation of spontaneous accumulation of NFATcl in the nuclei of resting fibers. It is clear that studies using only a few particular skeletal muscles risk overlooking interesting phenomena. Given the complexity of transcriptional regulation, the heterogeneous nature of the physiology and molecular biology of various skeletal muscles and the diverse tissues which surround them, we suspect that examining a wider variety of muscles specifically relevant to individual diseases in controlled in-vitro settings will prove fruitful.

Our culturing of intercostal muscles aims to do this not only by providing a particular model system specifically relevant to the pathology of COPD and the respiratory complications of the muscular dystrophies $[16,17]$, but also by improving the general techniques of adherent isolated muscle fiber culture. If these techniques are optimized for poorly adhering muscle types, generating cultures from any muscle in the body may become substantially easier, and using the most relevant tissue in a given disease model may become routine.

\section{Acknowledgment}

This work was supported by NIH-NIAMS Grants R01AR055099, R01-AR056477. P. Robison was supported by the Interdisciplinary Training Program Muscle Biology T32AR007592.

\section{References}

[1] R. Bottinelli and C. Reggiani, "Human skeletal muscle fibres: molecular and functional diversity," Progress in Biophysics and Molecular Biology, vol. 73, no. 2-4, pp. 195-262, 2000.

[2] R. I. Close, "Dynamic properties of mammalian skeletal muscles," Physiological Reviews, vol. 52, no. 1, pp. 129-197, 1972.

[3] J. C. Calderón, P. Bolaños, S. H. Torres, G. Rodríguez-Arroyo, and C. Caputo, "Different fibre populations distinguished by their calcium transient characteristics in enzymatically dissociated murine flexor digitorum brevis and soleus muscles," Journal of Muscle Research and Cell Motility, vol. 30, no. 3-4, pp. 125-137, 2009.

[4] J. C. Calderón, P. Bolaños, and C. Caputo, "Myosin heavy chain isoform composition and $\mathrm{Ca}^{2+}$ transients in fibres from enzymatically dissociated murine soleus and extensor digitorum longus muscles," Journal of Physiology, vol. 588, no. 1, pp. 267-279, 2010.

[5] R. L. Moss, G. M. Diffee, and M. L. Greaser, "Contractile properties of skeletal muscle fibers in relation to myofibrillar protein isoforms," Reviews of Physiology, Biochemistry \& Pharmacology, vol. 126, pp. 1-63, 1995.

[6] D. Pette and R. S. Staron, "Cellular and molecular diversities of mammalian skeletal muscle fibers," Reviews of Physiology Biochemistry and Pharmacology, vol. 116, pp. 1-76, 1990.

[7] S. Schiaffino and C. Reggiani, "Molecular diversity of myofibrillar proteins: gene regulation and functional significance," Physiological Reviews, vol. 76, no. 2, pp. 371-423, 1996.

[8] B. Polla, G. D’Antona, R. Bottinelli, and C. Reggiani, "Respiratory muscle fibres: specialisation and plasticity," Thorax, vol. 59, no. 9, pp. 808-817, 2004.

[9] A. De Troyer, P. A. Kirkwood, and T. A. Wilson, "Respiratory action of the intercostal muscles," Physiological Reviews, vol. 85, no. 2, pp. 717-756, 2005.

[10] A. Taylor, "The contribution of the intercostal muscles to the effort of respiration in man," The Journal of Physiology, vol. 151, pp. 390-402, 1960.

[11] D. Faithfull, J. G. Jones, and C. Jordan, "Measurement of the relative contributions of rib cage and abdomen/diaphragm to tidal breathing in man," British Journal of Anaesthesia, vol. 51, no. 5, pp. 391-398, 1979. 
[12] G. H. Koepke, E. M. Smith, A. J. Murphy, and D. G. Dickinson, "Sequence of action of the diaphragm and intercostal muscles during respiration. I. Inspiration," Archives of Physical Medicine and Rehabilitation, vol. 39, no. 7, pp. 426-430, 1958.

[13] A. J. Murphy, G. H. Koepke, E. M. Smith, and D. G. Dickinson, "Sequence of action of the diaphragm and intercostal muscles during respiration. II. Expiration," Archives of Physical Medicine and Rehabilitation, vol. 40, pp. 337-342, 1959.

[14] M. Mizuno and N. H. Secher, "Histochemical characteristics of human expiratory and inspiratory intercostal muscles," Journal of Applied Physiology, vol. 67, no. 2, pp. 592-598, 1989.

[15] M. Mizuno, N. H. Secher, and B. Saltin, "Fibre types, capillary supply and enzyme activities in human intercostal muscles," Clinical Physiology, vol. 5, no. 2, pp. 121-135, 1985.

[16] L. D. Calvert, T. M. McKeever, W. J. Kinnear, and J. R. Britton, "Trends in survival from muscular dystrophy in England and Wales and impact on respiratory services," Respiratory Medicine, vol. 100, no. 6, pp. 1058-1063, 2006.

[17] A. E. Emery, "The muscular dystrophies," Lancet, vol. 359, no. 9307, pp. 687-695, 2002.

[18] J. G. Gea, "Myosin gene expression in the respiratory muscles," European Respiratory Journal, vol. 10, no. 10, pp. 2404-2410, 1997.

[19] M. G. Klein, H. Cheng, L. F. Santana, Y. H. Jiang, W. J. Lederer, and M. F. Schneider, "Two mechanisms of quantized calcium release in skeletal muscle," Nature, vol. 379, no. 6564, pp. 455458, 1996.

[20] L. Kovacs, E. Rios, and M. F. Schneider, "Calcium transients and intramembrane charge movement in skeletal muscle fibres," Nature, vol. 279, no. 5712, pp. 391-396, 1979.

[21] S. L. Carroll, M. G. Klein, and M. F. Schneider, "Calcium transients in intact rat skeletal muscle fibers in agarose gel," American Journal of Physiology, vol. 269, no. 1, pp. C28-C34, 1995.

[22] S. L. Carroll, M. G. Klein, and M. F. Schneider, "Decay of calcium transients after electrical stimulation in rat fast- and slow-twitch skeletal muscle fibres," Journal of Physiology, vol. 501, no. 3, pp. 573-588, 1997.

[23] Y. Liu, S. L. Carroll, M. G. Klein, and M. F. Schneider, "Calcium transients and calcium homeostasis in adult mouse fast-twitch skeletal muscle fibers in culture," American Journal of Physiology, vol. 272, no. 6, pp. C1919-C1927, 1997.

[24] Y. Liu, Z. Cseresnyés, W. R. Randall, and M. F. Schneider, "Activity-dependent nuclear translocation and intranuclear distribution of NFATc in adult skeletal muscle fibers," Journal of Cell Biology, vol. 155, no. 1, pp. 27-39, 2001.

[25] B. L. Prosser, N. T. Wright, E. O. Hernãndez-Ochoa et al., "S100A1 binds to the calmodulin-binding site of ryanodine receptor and modulates skeletal muscle excitation-contraction coupling," Journal of Biological Chemistry, vol. 283, no. 8, pp. 5046-5057, 2008.

[26] L. D. Brown, G. G. Rodney, E. Hernández-Ochoa, C. W. Ward, and M. F. Schneider, " $\mathrm{Ca}^{2+}$ sparks and T tubule reorganization in dedifferentiating adult mouse skeletal muscle fibers," American Journal of Physiology. Cell Physiology, vol. 292, no. 3, pp. C1156-C1166, 2007.

[27] L. D. Brown and M. F. Schneider, "Delayed dedifferentiation and retention of properties in dissociated adult skeletal muscle fibers in vitro," In Vitro Cellular and Developmental Biology, vol. 38, no. 7, pp. 411-422, 2002.

[28] T. Ploug, B. van Deurs, H. Ai, S. W. Cushman, and E. Ralston, "Analysis of GLUT4 distribution in whole skeletal muscle fibers: identification of distinct storage compartments that are recruited by insulin and muscle contractions," Journal of Cell Biology, vol. 142, no. 6, pp. 1429-1446, 1998.

[29] G. R. Crabtree and E. N. Olson, "NFAT signaling: choreographing the social lives of cells," Cell, vol. 109, no. 2, supplement 1, pp. S67-S79, 2002.

[30] A. Rao, C. Luo, and P. G. Hogan, "Transcription factors of the NFAT family: regulation and function," Annual Review of Immunology, vol. 15, pp. 707-747, 1997.

[31] S. M. Cardoso, P. Mutch, P. J. Scotting, and P. M. Wigmore, "Gene transfer into intact fetal skeletal muscle grown in vitro," Muscle and Nerve, vol. 30, no. 1, pp. 87-94, 2004.

[32] S. G. Cull-Candy, R. Miledi, and O. D. Uchitel, "Denervation changes in normal and myasthenia gravis human muscle fibres during organ culture," Journal of Physiology, vol. 333, pp. 227249, 1982.

[33] R. L. Ruff and D. Whittlesey, " $\mathrm{Na}^{+}$current densities and voltage dependence in human intercostal muscle fibres," Journal of Physiology, vol. 458, pp. 85-97, 1992.

[34] L. Ziskind-Conhaim and M. J. Dennis, "Development of rat neuromuscular junctions in organ culture," Developmental Biology, vol. 85, no. 1, pp. 243-251, 1981.

[35] L. Ziskind-Conhaim, I. Geffen, and Z. W. Hall, "Redistribution of acetylcholine receptors on developing rat myotubes," Journal of Neuroscience, vol. 4, no. 9, pp. 2346-2349, 1984.

[36] K. Yamanouchi, T. Hosoyama, Y. Murakami, S. Nakano, and M. Nishihara, "Satellite cell differentiation in goat skeletal muscle single fiber culture," Journal of Reproduction and Development, vol. 55, no. 3, pp. 252-255, 2009. 

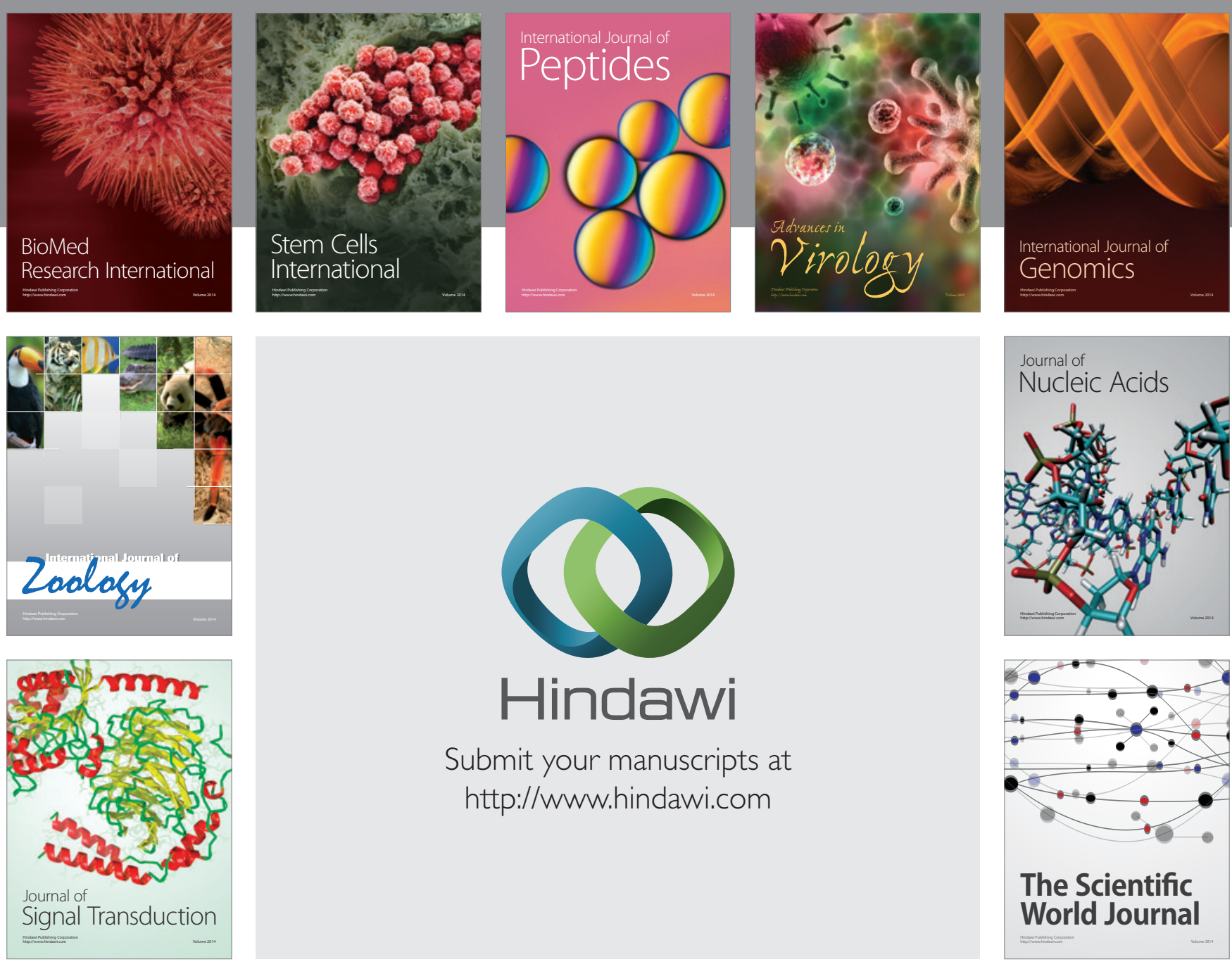

Submit your manuscripts at

http://www.hindawi.com
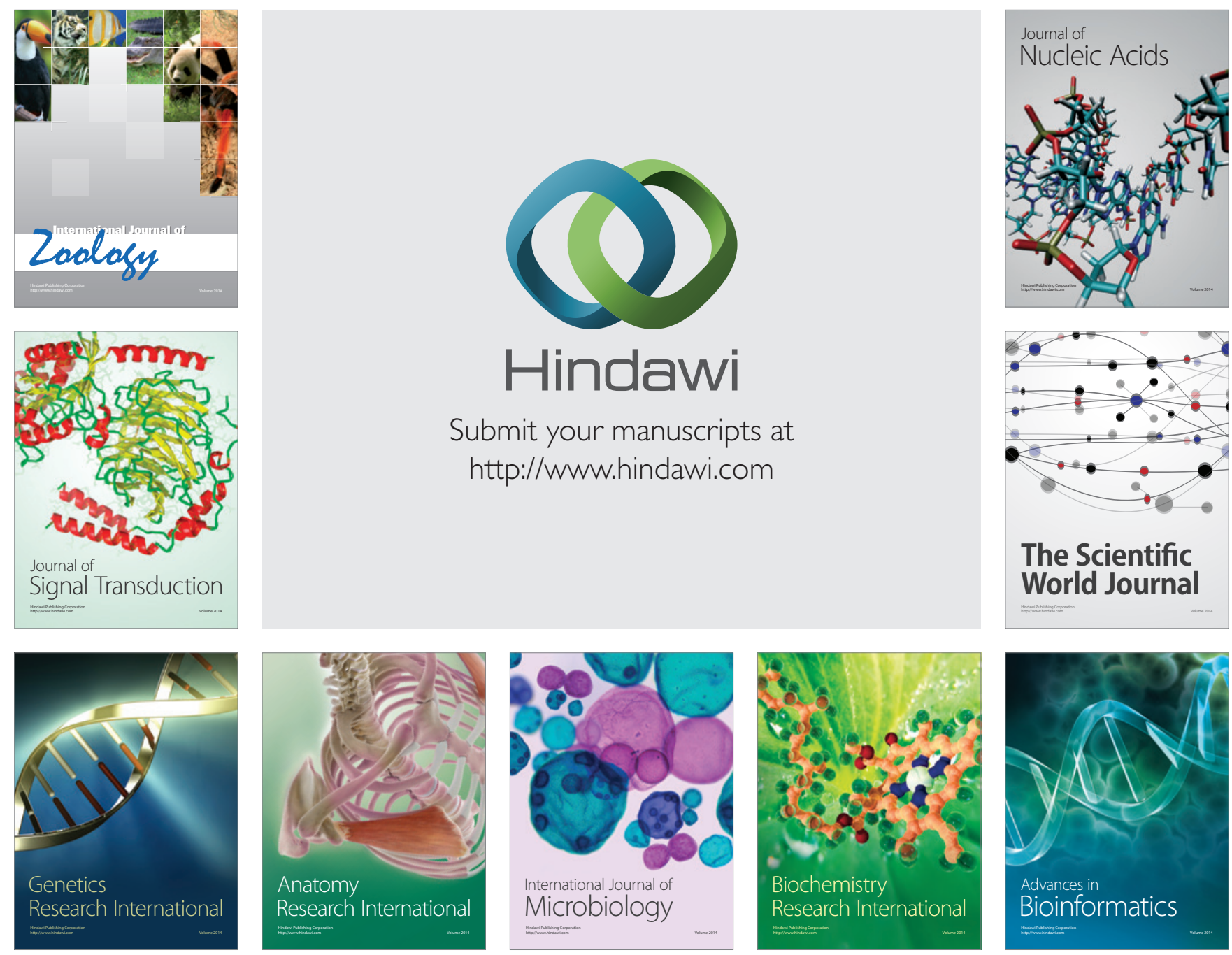

The Scientific World Journal
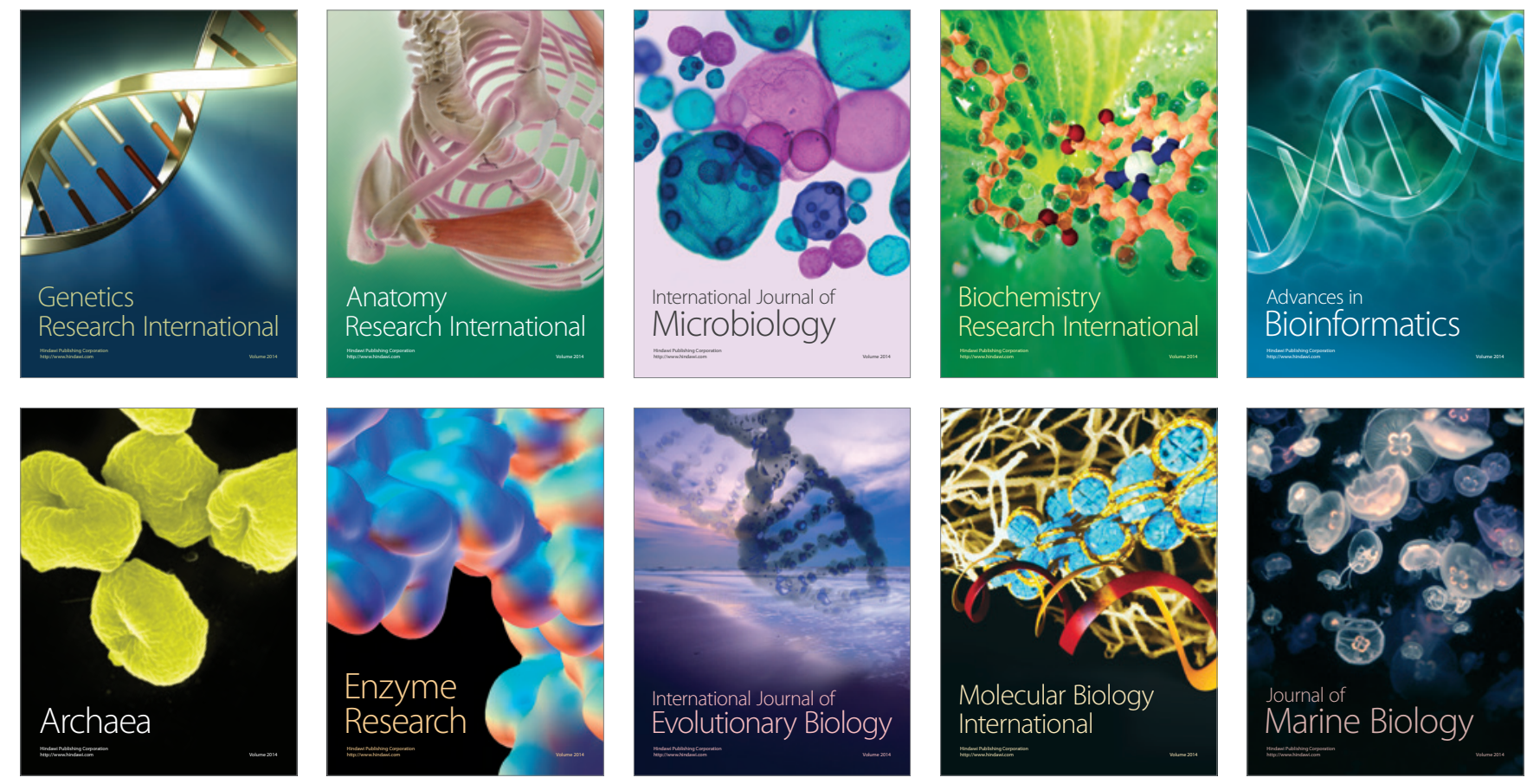